\title{
Metformin and Sildenafil Attenuate Inflammation and Suppress Apoptosis After Ischemia/Reperfusion Injuries in Rat Urinary Bladder
}

\author{
Jong Mok Park ${ }^{1, \star}$, Ju Hyun Shin ${ }^{2, *}$, Seung Woo Yang², Ji Yong Lee², Chung Lyul Lee², Jae Sung Lim², Ki Hak Song², \\ Gun Hwa Kim ${ }^{3}$, Yong Gil Na ${ }^{1}$ \\ ${ }^{1}$ Department of Urology, Chungnam National University Sejong Hospital, Chungnam National University School of Medicine, Sejong, Korea \\ ${ }^{2}$ Department of Urology, Chungnam National University Hospital, Chungnam National University School of Medicine, Daejeon, Korea \\ ${ }^{3}$ Research Center for Bioconvergence Analysis, Korea Basic Science Institute, Cheongju, Korea
}

Purpose: Although metformin and sildenafil can protect various organs against ischemia/reperfusion (I/R) injuries, their effects and mechanisms of action in bladder I/R injuries remain unknown. This study investigated the effects and mechanisms of action of metformin and sildenafil against bladder $\mathrm{I} / \mathrm{R}$ insults in rats.

Methods: One hundred male Sprague-Dawley rats were randomly divided into 5 groups, each of which contained 20 rats: a sham-operated group, a bladder I/R group, and bladder I/R groups treated with metformin, sildenafil, or both agents. Ischemia was induced by clamping the bilateral common iliac arteries with atraumatic vascular clamps for 2 hours, followed by reperfusion for 7 days. During this period, rats were injected once daily with 4-mg/kg metformin and/or 1-mg/kg sildenafil. Results: I/R injuries induced increased malondialdehyde levels and myeloperoxidase activity and decreased superoxide dismutase activity. These changes were attenuated by treatment with metformin and/or sildenafil. The $\mathrm{I} / \mathrm{R}$ group had significantly higher Jun N-terminal kinase, p38 mitogen-activated protein kinase (MAPK), Bax, caspase-3, and nuclear factor-kappa B (NF- $\mathrm{kB}$ ) levels, and lower extracellular signal-regulated kinase, and Bcl-2 levels in the bladder than the sham-operated group; these changes were significantly ameliorated by metformin and/or sildenafil treatment. No differences in the levels of these markers were observed between rats coadministered metformin and sildenafil and those treated with either agent alone.

Conclusions: Metformin and sildenafil protected the rat bladder against I/R injuries. This effect may have been due to the inhibition of reactive oxygen species production through MAPK, Bax, and Bcl-2 activation, and the restoration of inflammation through NF-kB inhibition. However, the combination of metformin and sildenafil was not more effective than either agent alone.

Keywords: Ischemia-reperfusion; Metformin; Urinary bladder

- Fund/Grant Support: This research was supported by the research fund of Chungnam National University.

- Research Ethics: All animal experiments were performed according to guidelines of the ethics committee of Chungnam National University and the Institutional Animal Care and Use Committee (approval number: CNU-01294).

- Conflict of Interest: No potential conflict of interest relevant to this article was reported.

Corresponding author: Yong Gil Na (i) https://orcid.org/0000-0002-0794-5459 Department of Urology, Chungnam National University Sejong Hospital, Chungnam National University School of Medicine, 20 Bodeum 7-ro, Sejong 30099, Korea

Email: yongna@cnu.ac.kr

${ }^{\star}$ Jong Mok Park and Ju Hyun Shin contributed equally to this study as co-first authors.

Submitted: July 1, 2021 / Accepted after revision: September 3, 2021 


\begin{abstract}
- HIGHLIGHTS
- Metformin and sildenafil protected the rat bladder against I/R injuries.

- This effect may have been due to the inhibition of reactive oxygen species production through MAPK, Bax, and Bcl-2 activation, and the restoration of inflammation through NF-KB inhibition.

-The combination of metformin and sildenafil was not more effective than either agent alone.
\end{abstract}

\section{INTRODUCTION}

Bladder dysfunction and associated lower urinary tract symptoms (LUTS) are common in elderly men and women, and men are slightly more vulnerable [1]. LUTS in men are thought to be caused by mechanical obstruction of the bladder outlet induced by hypertrophic prostate enlargement [2]. Repeated hypoxia and ischemia/reperfusion (I/R) injuries related to voiding dysfunction due to bladder outlet obstruction induce the production of free radicals, leading to the generation of reactive oxygen species (ROS) in the bladder wall [3,4]. ROS formation causes age-associated damage to cells and organs, and reduces levels of antioxidant enzymes, thereby inducing LUTS [5].

Metformin is an antihyperglycemic drug that is frequently used to treat type 2 diabetes mellitus and can reduce ROS levels [6]. Metformin attenuates the activation of mitochondria and activates AMP-activated protein kinase (AMPK) [7]. AMPK is a key modulator of cellular energy regulation, and activated AMPK changes anabolic cells to a catabolic state. Recent studies have shown that activation of AMPK by metformin attenuates the expression of $\mathrm{p} 38$ mitogen-activated protein kinase (p38 MAPK) and induces the expression of extracellular signalregulated kinase 3 (ERK3) [8]. AMPK signaling can also reversibly regulate hyperactive MAPK signaling in cancer cells by phosphorylating its key components, RAF/KSR family kinases [9]. Metformin also has favorable effects on cardiovascular disease; metformin treatment decreases infarct size during I/R injuries in rats [10]. Low doses of metformin may ameliorate renal I/R injuries by increasing the energy supply to hypoxic organs and decreasing the activity of inflammation-related factors [11]. However, it is unclear whether metformin treatment after $\mathrm{I} / \mathrm{R}$ injuries can prevent ROS activity in bladder tissue.

Phosphodiesterase 5 (PDE5) inhibitors such as sildenafil were developed to manage angina pectoris and subsequently have been used to treat patients with erectile dysfunction [12]. Sildenafil ameliorates vascular dysfunction, has been found to decrease the infarct area in an animal model of myocardial infarction [13], and has shown reno-protective efficacy in models of renal injury [14]. A PDE5 inhibitor was reported to increase blood flow to the pelvic organs, thereby improving bladder function, in a rat model [15]. However, the mechanisms of action of PDE5 inhibitors on LUTS associated with I/R injuries, as well as the drug interactions between metformin and PDE5 inhibitors in the bladder, remain to be determined.

The present study was therefore designed to investigate the protective effects and mechanisms of metformin, sildenafil, and coadministration of both drugs on $\mathrm{I} / \mathrm{R}$ injuries in the urinary bladder in rats.

\section{MATERIALS AND METHODS}

\section{Animal Model and Experimental Groups}

A total of 100 male Sprague-Dawley rats, 12 weeks of age and weighing 250-300 g, were obtained from Samtako Bio Korea (Osan, Korea) and housed in separate cages under a controlled 12-hour light $/ 12$-hour dark cycle at $23^{\circ} \mathrm{C}$. Animals were fed standard rat chow and had free access to tap water. All procedures were conducted in accordance with the guidelines of the ethics committee and the Institutional Animal Care and Use Committee of Chungnam National University.

The 100 rats were randomly divided into 5 groups, each of which contained 20 rats: a sham-operated group, a bladder $\mathrm{I} / \mathrm{R}$ group, and bladder $\mathrm{I} / \mathrm{R}$ groups treated with metformin, sildenafil, or both agents. Rats in the sham-operated group were subjected to all surgical procedures, except for vascular clamping, and rats in the $\mathrm{I} / \mathrm{R}$ group were subjected to $\mathrm{I} / \mathrm{R}$ injuries. The animals in the sham-operated and I/R groups were injected intraperitoneally with $0.9 \%$ saline solution once daily for 7 days. Rats in the I/R plus metformin, I/R plus sildenafil, and I/R plus metformin and sildenafil groups were subjected to $I / R$ injuries, followed by intraperitoneal injection of $4-\mathrm{mg} / \mathrm{kg}$ metformin [16], 1-mg/kg sildenafil [17], or 4-mg/kg metformin and 1-mg/ $\mathrm{kg}$ sildenafil, respectively, in $0.9 \%$ saline once daily for 7 days.

\section{In vivo Experimental Protocol}

All surgical procedures were performed under anesthesia, which was induced by intramuscular injection of xylazine (Rompun, $10 \mathrm{mg} / \mathrm{kg}$ ) and ketamine (Imalgene, $100 \mathrm{mg} / \mathrm{kg}$ ). A lower 
abdominal vertical incision was made. Ischemia was induced by clamping the bilateral common iliac arteries with atraumatic clamps for 2 hours. Following clamp removal, the incision site was sutured. Sham operations were conducted using a similar method, except the arteries were not clamped. During the 7-day reperfusion period, the rats were injected once daily with saline or metformin and/or sildenafil. At the end of the reperfusion period, the rats were euthanized using carbon dioxide gas. The bladders were harvested, cleared of adhering connective tissue, and immediately stored at $-80^{\circ} \mathrm{C}$ or fixed in $10 \%$ phosphate-buffered formalin.

\section{RT-PCR Assay}

Sequences of interest were amplified from cDNA by polymerase chain reaction (PCR). Amplification consisted of an initial denaturation phase for 5 minutes at $95^{\circ} \mathrm{C}$, followed by 35 cycles of denaturation for 30 seconds at $95^{\circ} \mathrm{C}$, annealing at $58^{\circ} \mathrm{C}$ for 30 seconds, and extension at $72^{\circ} \mathrm{C}$ for 30 seconds, and a final extension at $72^{\circ} \mathrm{C}$ for 5 minutes. PCR products were analyzed by electrophoresis on a $1.2 \%$ agarose gel. The primer sequences are illustrated in Supplementary Table 1.

\section{Western Blot Assay}

Frozen bladder tissues were homogenized in Pro-Prep protein extraction solution (Intron, Daejeon, Korea). Proteins were analyzed by $12 \%$ sodium dodecyl sulfate-polyacrylamide gel electrophoresis and transferred to polyvinylidene fluoride membranes. The primary antibodies used are listed in Supplementary Table 2 . The membranes were washed in phosphate-buffered saline $(0.1 \%$ Tween), followed by incubation with peroxidaselabeled secondary antibodies (Supplementary Table 2). Immune-labeled proteins were detected using a SuperSignal enhanced chemiluminescence kit (Thermo Fisher, Waltham, MA, USA) and quantitated using a Western blot detection system (Bio-Rad, Hercules, CA, USA), with the density of each band normalized to that of GAPDH.

\section{Immunohistochemical Analysis of Caspase-3 Activity}

Rat bladder tissue samples were immediately fixed in formalin, dehydrated in graded ethanol, cleared in xylene, and embedded in paraffin blocks using automatic processing and embedding equipment (Tissue Tek VIP; Sakura Finetek, Torrance, CA, USA). The paraffin-embedded tissue samples were sectioned, and then sections were deparaffinized in xylene and rehydrated using a series of increasing ethanol concentrations. The sections were incubated overnight with an anti-caspase-3 antibody (diluted 1:500, Cell Signaling Technology, Danvers, MA, USA), followed by washing and incubation with secondary antibodies (EnVision+ System HRP-labeled polymer anti-rabbit, Dako; Agilent Technologies, Santa Clara, CA, USA). The sections were developed by incubation in diaminobenzidine tetrachloride solution and counterstained with Mayer's hematoxylin.

\section{Biochemical Analysis of Enzyme Activity}

Superoxide dismutase (SOD; Abcam, Cambridge, UK) activity, reported as $\mathrm{U} / \mathrm{mg}$ tissue, was determined by measuring enzyme-mediated inhibition of nitroblue tetrazolium reduction [18]. One unit of SOD was defined as the enzyme activity that inhibited nitroblue tetrazolium reduction by $50 \%$. Malondialdehyde (MDA; Abcam, Cambridge, UK) levels in bladder tissues, expressed as nmol/g tissue, were analyzed using a method based on the reaction of MDA with thiobarbituric acid at $95^{\circ} \mathrm{C}$ [19], which produces a pink pigment with maximum absorption at $532 \mathrm{~nm}$. Bladder injury was quantified by measuring myeloperoxidase (MPO; Abcam, Cambridge, UK) activity (expressed as $\mathrm{U} / \mathrm{g}$ ) after adding $\mathrm{O}$-dianisidine dihydrochloride and hydrogen peroxide [20].

\section{Statistical analysis}

All data were statistically analyzed using SPSS ver. 18.0 (SPSS Inc., Chicago, IL, USA). Data are expressed as mean \pm standard error of the mean. Three or more groups were compared using the Kruskal-Wallis test, and the P-value was adjusted with the Bonferroni correction. A P-value $<0.05$ was considered to indicate statistical significance.

\section{RESULTS}

\section{ERK1, JNK1, and p38 MAPK Expression}

We first assessed whether treatment with metformin or sildenafil could modulate the intracellular MAPK signaling pathway in bladder tissue after I/R injuries. The mRNA and protein expression levels of key molecules in this signaling pathway, ERK1, Jun N-terminal kinase 1 (JNK1), and p38 MAPK, were investigated using reverse-transcription PCR (RT-PCR) and Western blotting, respectively.

Tissue samples from the I/R group had significantly higher levels of JNK1 and p38 mRNA and significantly lower levels of ERK1 mRNA than bladder tissue from the sham-operated group (Fig. 1A). Treatment of I/R-subjected rats with metfor- 

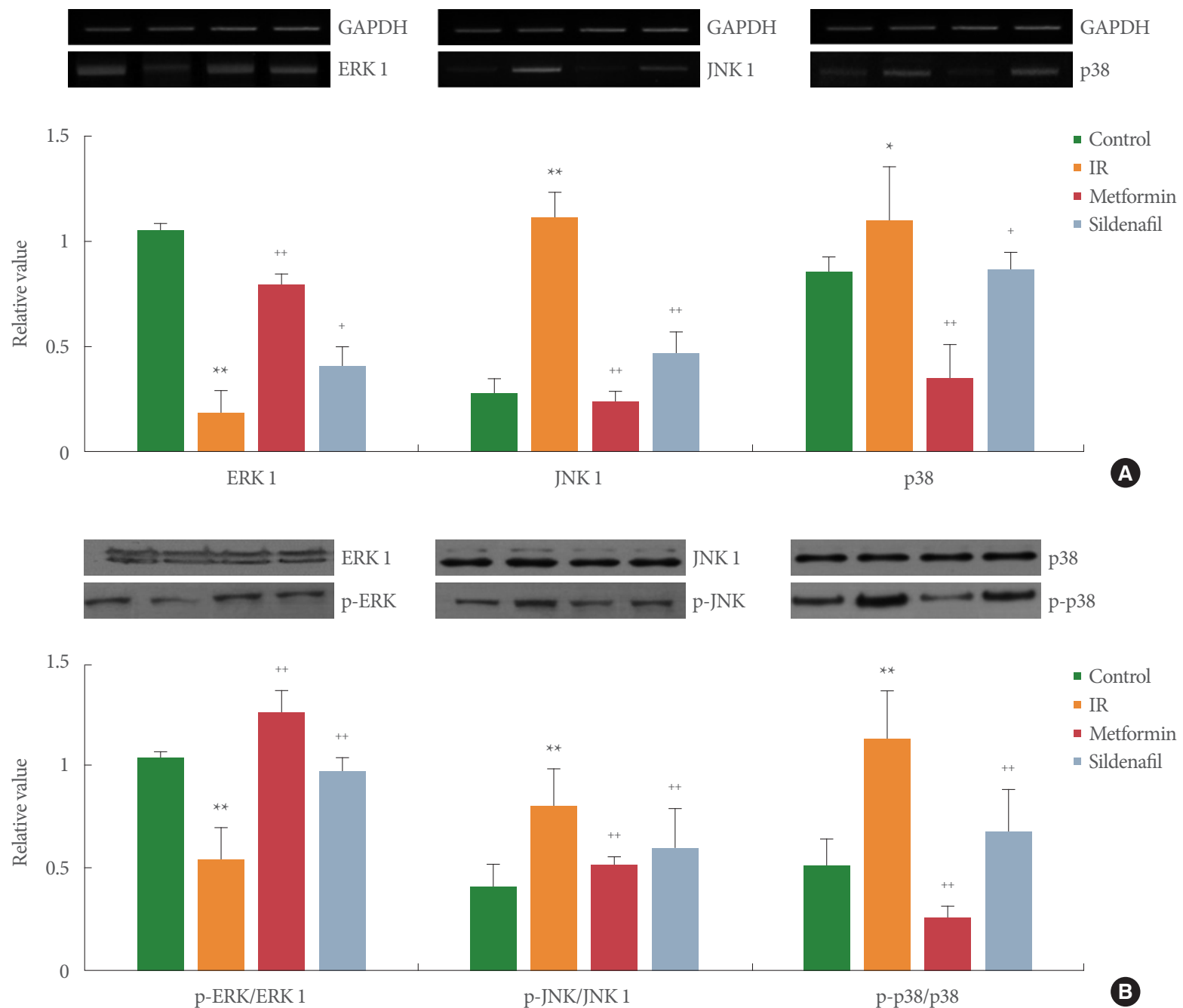

$\mathrm{p}-J \mathrm{NK} / J \mathrm{NK} 1$

p-p38/p38

B

Fig. 1. The effects of metformin and sildenafil on mitogen-activated protein kinase expression in bladder tissue. (A) Images and graphs of reverse-transcription polymerase chain reaction analyses of relative levels of extracellular signal-regulated kinase (ERK), Jun $\mathrm{N}$-terminal kinase (JNK), and p38 mRNAs in bladder tissue samples. (B) Images and graphs of Western blot analyses of relative levels of ERK, JNK, and p38 proteins in bladder tissue. Results presented as mean \pm standard error of the mean. p-ERK, phospho-ERK; pJNK, phospho-JNK. ${ }^{*} \mathrm{P}<0.05,{ }^{* *} \mathrm{P}<0.01$ compared with the sham-operated group. ${ }^{\dagger} \mathrm{P}<0.05$, ${ }^{*} \mathrm{P}<0.01$ compared with the ischemia/ reperfusion (I/R) group.

min or sildenafil significantly reduced the expression of JNK1 and p38 mRNA and significantly enhanced the expression of ERK1 mRNA.

Western blotting analysis showed that phospho-JNK1(pJNK1) and p-p38 MAPK levels were higher, and phosphoERK1(p-ERK1) levels were lower, in bladder tissue samples from I/R-subjected rats than in samples from sham-operated rats (Fig. 1B). The treatment of I/R-subjected rats with metformin or sildenafil significantly reduced the levels of p-JNK1 and p-p38, and increased the level of p-ERK1.

\section{Bax and Bcl-2 Expression}

The RT-PCR analysis showed that the level of Bax mRNA was significantly higher, the level of Bcl-2 mRNA was significantly lower, and the ratio of Bax/Bcl-2 mRNA was significantly higher in the $\mathrm{I} / \mathrm{R}$ group than in the sham-operated group (Fig. 2A). Treatment of I/R-subjected rats with metformin or sildenafil significantly increased the level of Bcl-2 mRNA and the Bax/ Bcl-2 mRNA ratio, and significantly reduced the level of Bax mRNA.

Western blotting assays of Bcl-2 and Bax protein expression 

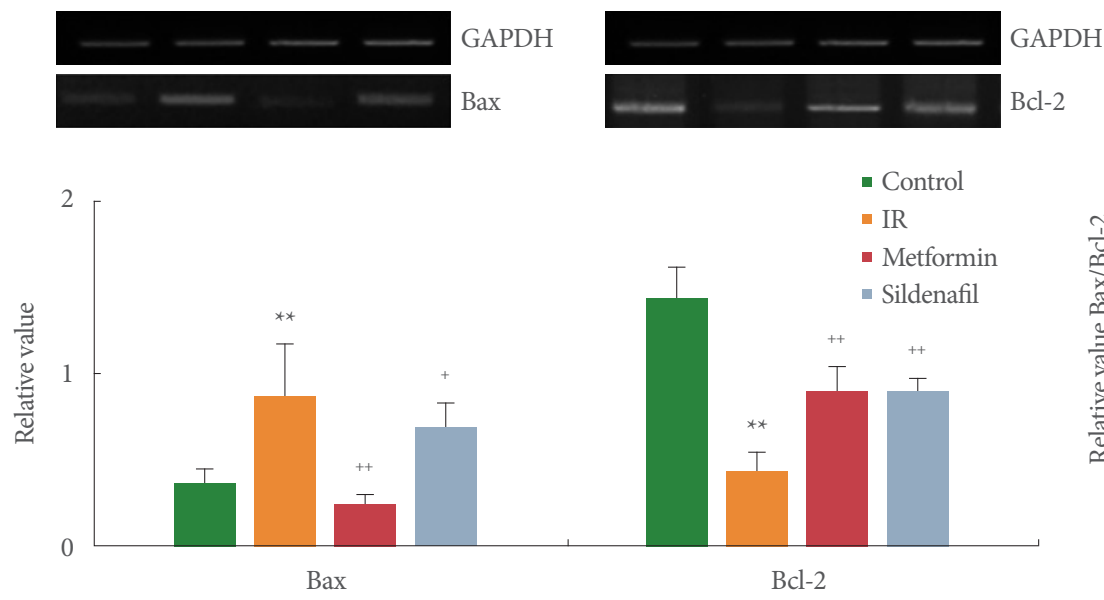

Bcl-2

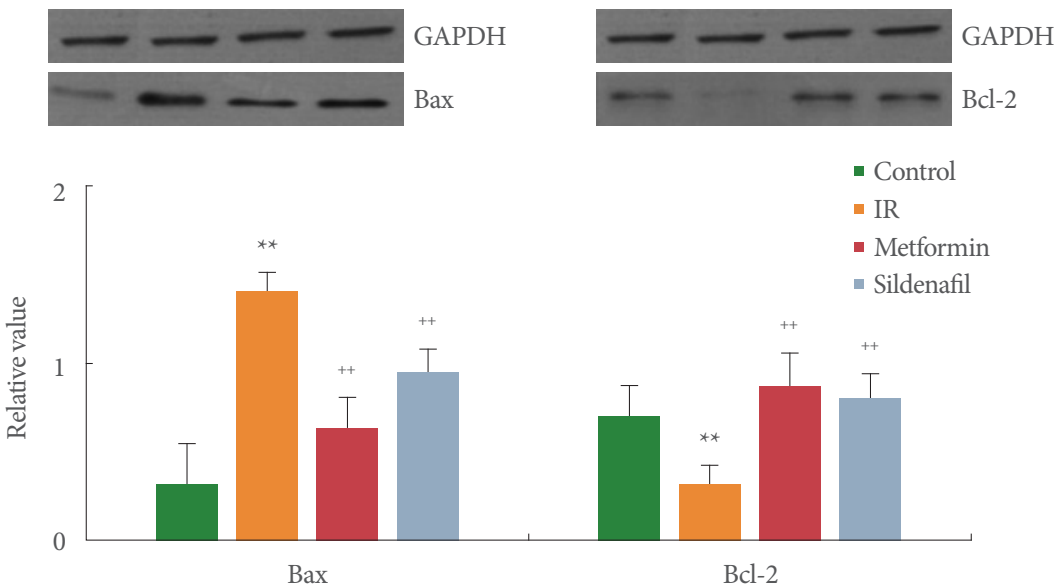

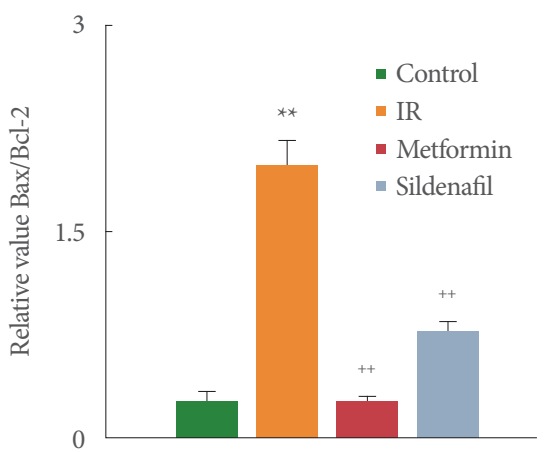

Bax/Bcl-2

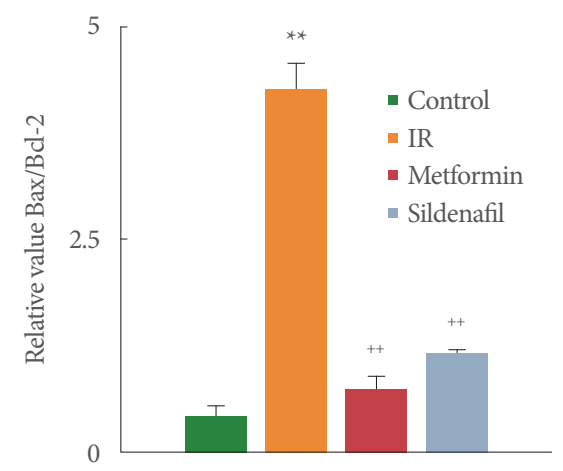

$\mathrm{Bax} / \mathrm{Bcl}-2$

Fig. 2. The effects of metformin and sildenafil on Bax and Bcl-2 expression in bladder tissue. (A) Image and graphs of reverse-transcription polymerase chain reaction (RT-PCR) analysis of the relative expression of Bax and Bcl-2 mRNA and the Bax/Bcl-2 mRNA ratio in bladder tissue samples. (B) Images and graphs of Western blot analysis of the relative expression of Bax and Bcl-2 proteins and the $\mathrm{Bax} / \mathrm{Bcl}-2$ protein ratio in bladder tissue samples. Results are presented as mean \pm standard error of the mean. ${ }^{\star} \mathrm{P}<0.05,{ }^{*} \mathrm{P}<0.01$ compared with the sham-operated group. ${ }^{\dagger} \mathrm{P}<0.05,{ }^{*} \mathrm{P}<0.01$ compared with the ischemia/reperfusion (I/R) group.

showed that the level of Bcl-2 protein was significantly lower, and the level of Bax protein expression and the Bax/Bcl-2 ratio were significantly higher, in the $\mathrm{I} / \mathrm{R}$ group than in the sham-operated group (Fig. 2B). I/R-subjected rats treated with metformin and I/R-subjected rats treated with sildenafil had significantly higher levels of Bcl-2 protein expression, significantly higher Bax/Bcl-2 ratios, and significantly lower levels of Bax protein expression than I/R-subjected rats.

\section{NF-kB Expression}

RT-PCR analysis showed that the level of NF- $\kappa B$ mRNA was significantly higher in the I/R group than in the sham-operated group (Fig. 3). Sildenafil and metformin significantly reduced the level of NF- $\mathrm{B}$ mRNA. Western blotting analysis showed similar results; I/R injury increased the expression of NF- $\mathrm{KB}$ protein, and treatment with metformin or sildenafil reduced the I/R-mediated increase in NF- $\kappa \mathrm{B}$ protein expression. In addition, metformin reduced the I/R-induced increase in NF- $\mathrm{kB}$ expression to a greater extent than sildenafil in both RT-PCR and Western blotting analyses $(\mathrm{P}<0.05)$.

\section{The Effects of Coadministering Metformin and Sildenafil}

The effects of coadministering metformin and sildenafil on mRNA and protein levels were compared with those of metformin only by RT-PCR and western blotting experiments (Fig. 4). RT-PCR analysis of mRNAs encoding 7 proteins (ERK, JNK, 


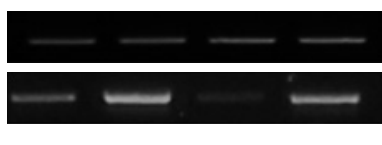
GAPDH

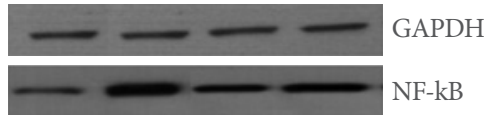

\section{A}

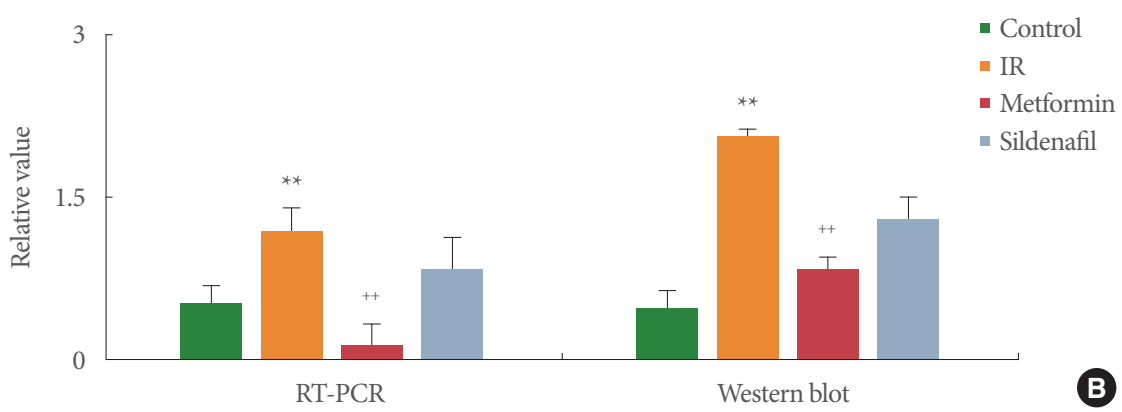

Fig. 3. The effects of metformin and sildenafil on nuclear factor-kappa B (NF- $\kappa B$ ) expression in bladder tissue. (A) Representative images of reverse-transcription polymerase chain reaction (RT-PCR) and Western blotting analyses of NF- $\kappa \mathrm{B}$ expression. (B) Graphs of RT-PCR and Western blot analyses of the relative expression of NF- $\kappa$ B mRNA and protein. Results are presented as mean \pm standard error of the mean. ${ }^{* *} \mathrm{P}<0.01$ compared with the sham-operated group. ${ }^{\star} \mathrm{P}<0.01$ compared with the ischemia/reperfusion (I/R) group.
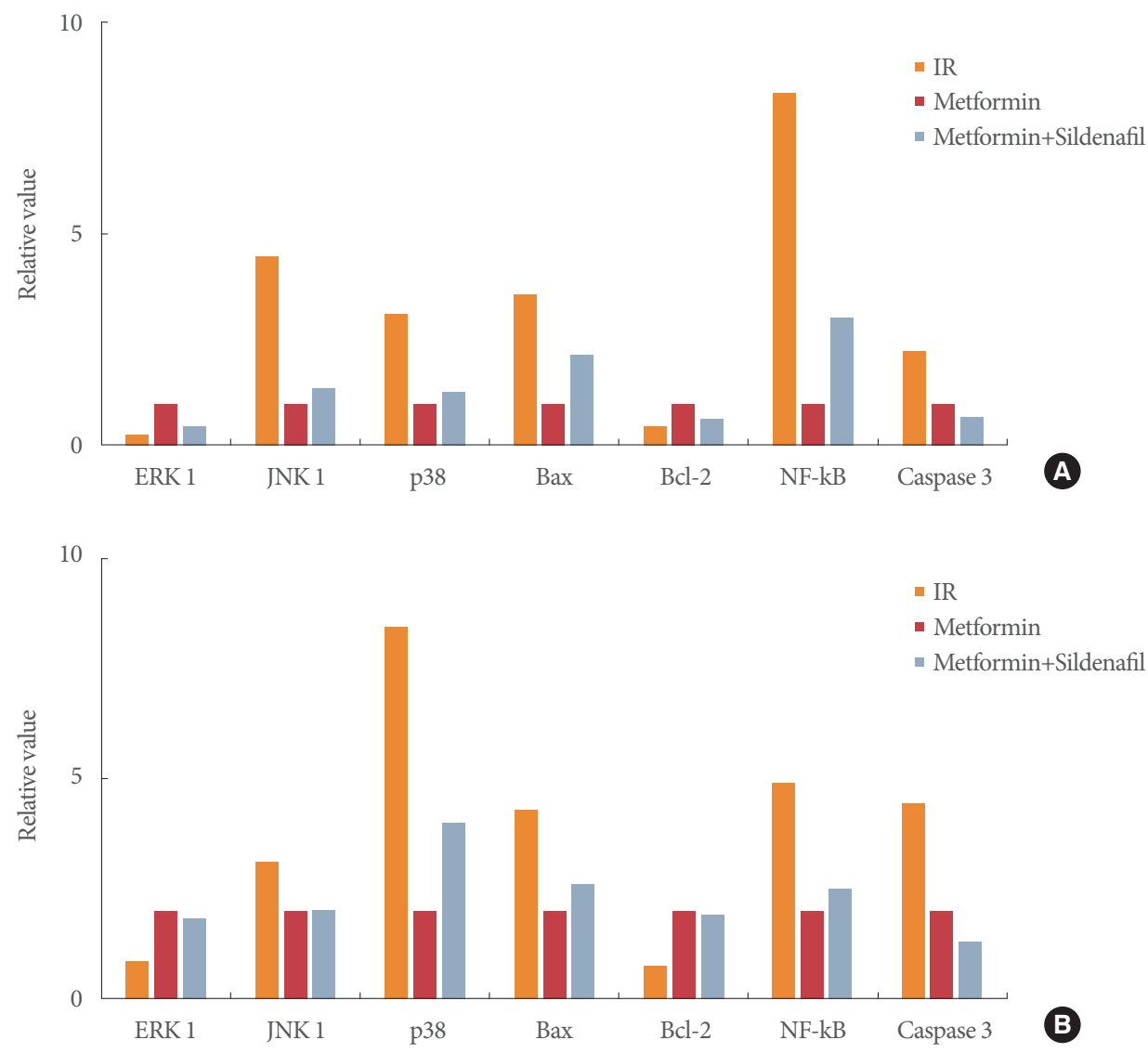

Fig. 4. The relative expression of ERK, JNK, p38, Bax, Bcl-2, NF- $\kappa$ B, and caspase-3. (A) mRNA levels (analyzed by reverse-transcription polymerase chain reaction) and (B) protein levels (analyzed by Western blotting) in bladder tissues of ischemia/reperfusion-subjected rats treated with metformin or metformin and sildenafil. The expression of 7 factors was analyzed. ERK, extracellular signalregulated kinase; JNK, Jun N-terminal kinase; NF- $\kappa B$, nuclear factor-kappa B; IR, ischemia/reperfusion. 
Table 1. The activity of superoxide dismutase (SOD), malondialdehyde (MDA), and myeloperoxidase (MDA) in the 5 groups of rats

\begin{tabular}{lccc}
\hline Group & MDA $(\mathrm{nM})$ & MPO $(\mathrm{U} / \mathrm{g}$ protein $)$ & SOD (\%) \\
\hline Control & $0.068 \pm 0.0073$ & $0.418 \pm 0.089$ & $87.43 \pm 0.96$ \\
I/R & $0.120 \pm 0.0059$ & $0.671 \pm 0.053$ & $49.69 \pm 1.44$ \\
Metformin & $0.074 \pm 0.0089$ & $0.350 \pm 0.031$ & $75.08 \pm 0.78$ \\
Sildenafil & $0.072 \pm 0.0036$ & $0.276 \pm 0.024$ & $73.07 \pm 0.63$ \\
Metformin plus sildenafil & $0.08 \pm 0.0027$ & $0.336 \pm 0.010$ & $66.94 \pm 0.33$ \\
\hline
\end{tabular}

Values are presented as mean \pm standard error of the mean.

$\mathrm{I} / \mathrm{R}$, ischemia/reperfusion.
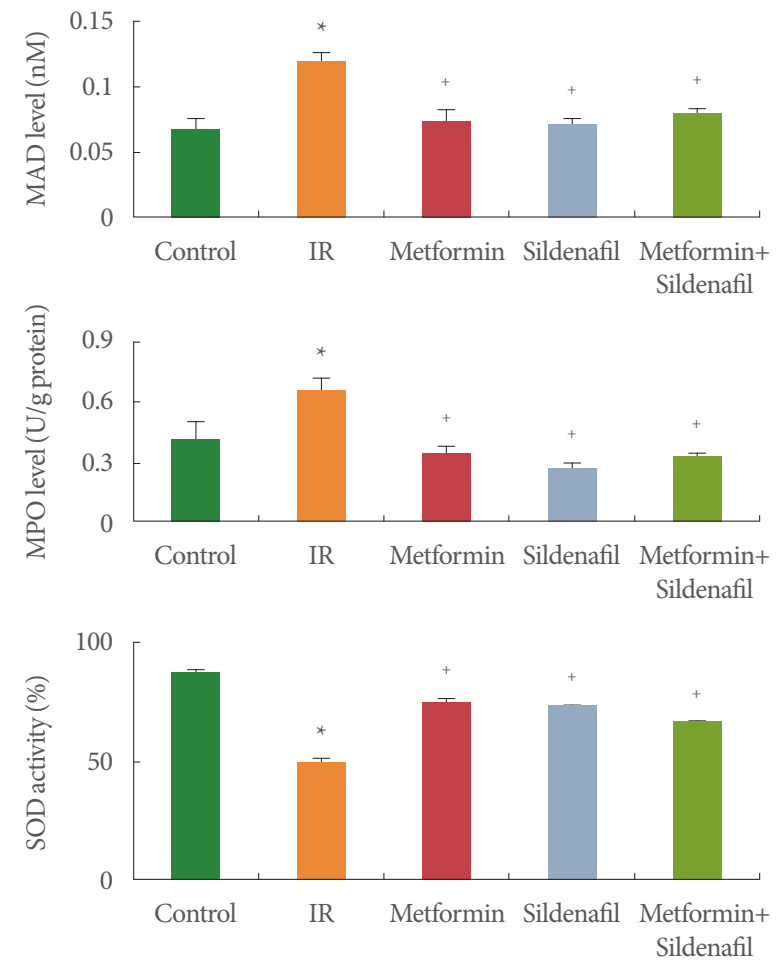

Fig. 5. The activity of malondialdehyde (MDA), myeloperoxidase (MPO), and superoxide dismutase (SOD) enzymes in the 5 groups of rats. ${ }^{\star} \mathrm{P}<0.05$ compared with the control group. ${ }^{\dagger} \mathrm{P}<$ 0.05 compared with the ischemia/reperfusion (I/R) group.

p38, Bax, Bcl-2, NF-kB, and caspase-3) showed that coadministration of metformin and sildenafil significantly altered the level of caspase-3 mRNA, but did not alter the expression of the other 6 mRNAs. Western blotting analysis yielded similar results; coadministration of metformin and sildenafil significantly altered only the level of caspase- 3 protein. These results showed that coadministration of metformin and sildenafil did not synergistically affect the pathways associated with MAPKs, Bax/ Bcl-2, and NK- $\mathrm{kB}$ activation.

\section{The Effects of Metformin and Sildenafil on Oxidative Stress Parameters}

The mean levels of MDA and MPO, which are markers of lipid peroxidation levels, were significantly higher in the I/R group than in the sham-operated group (Fig. 5, Table 1). Treatment of I/R-subjected rats with sildenafil and/or metformin significantly decreased the levels of MDA and MPO. There was no significant difference between the activity of either enzyme in I/Rsubjected rats coadministered metformin and sildenafil and I/ $\mathrm{R}$-subjected rats treated with metformin. SOD activity was significantly lower in the I/R group than in the sham-operated group. Treatment with metformin and/or sildenafil increased SOD activity. There was no significant difference in SOD activity between I/R-subjected rats co-administered metformin and sildenafil, and I/R-subjected rats treated with metformin.

\section{Caspase-3 Activation}

Apoptosis in the rat bladder was evaluated by immunohistochemically estimating the expression of cleaved caspase-3. Representative histological images from each group are shown in Fig. 6A, with the brown color indicating caspase-3 antibody staining in the cytoplasm of urothelial cells. Cleaved caspase- 3 levels were lowest in the sham-operated group and highest in the I/R group. Treatment of I/R-subjected rats with metformin and/or sildenafil significantly reduced the expression of cleaved caspase-3, although there was no significant difference between rats treated with both metformin and sildenafil and those treated with either agent alone.

Immunohistochemical findings were confirmed by RT-PCR and western blotting analyses (Fig. 6B). RT-PCR analysis showed that the expression of caspase- 3 mRNA was significantly higher in I/R-treated rats than in sham-operated rats. Metformin treatment significantly reduced caspase- 3 mRNA levels. Although sildenafil also reduced caspase- 3 mRNA expression slightly, this 

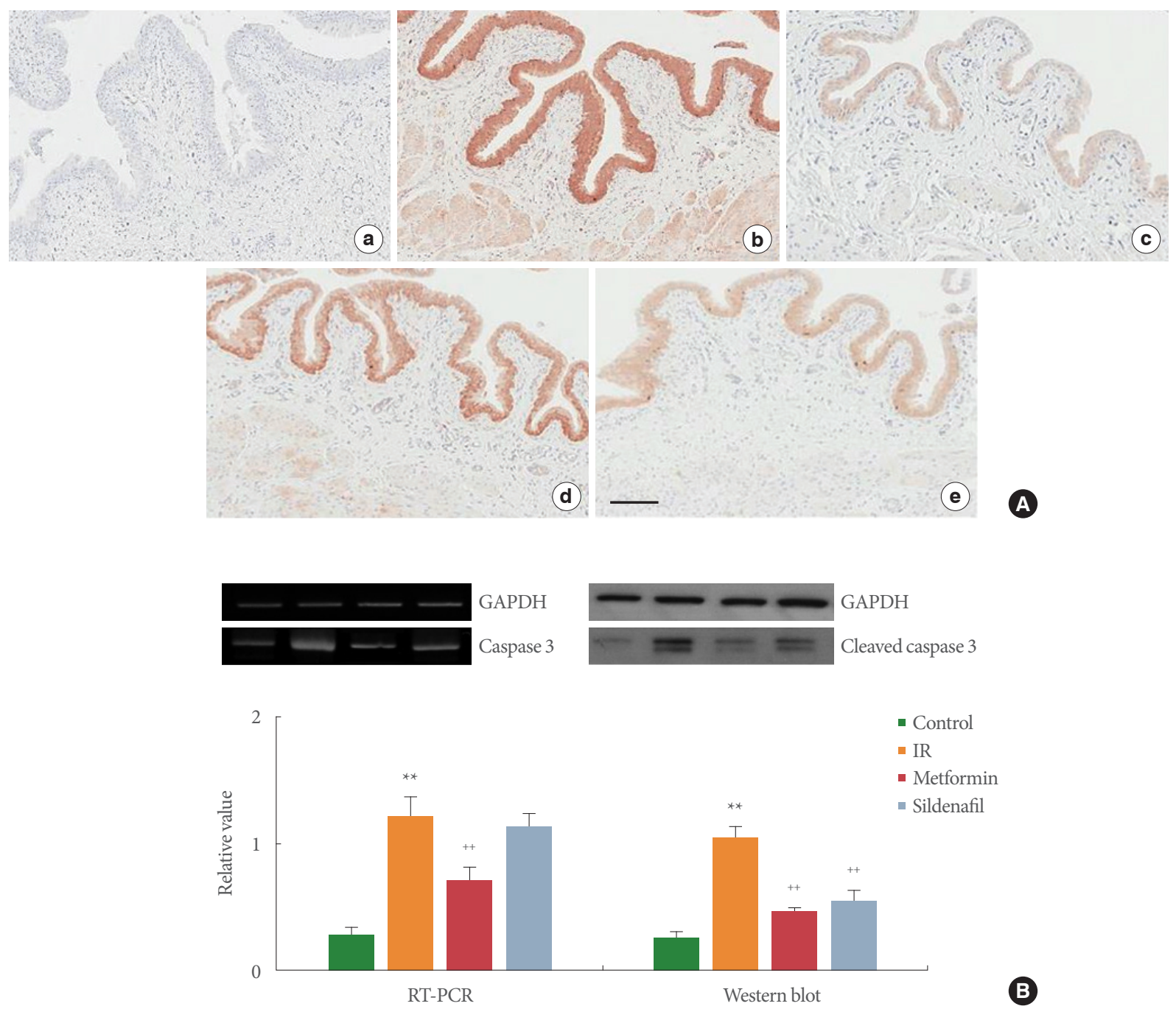

B

Fig. 6. The effects of metformin and sildenafil on the expression of caspase- 3 and cleaved caspase- 3 in bladder tissue. (A) Immunohistochemical analysis of cleaved caspase-3 expression in the sham-operated (a), ischemia/reperfusion (I/R) (b), I/R plus metformin (c), $\mathrm{I} / \mathrm{R}$ plus sildenafil (d), and I/R plus metformin and sildenafil (e) groups. The brown staining indicates caspase- 3 expression. The scale bar indicates $100 \mu \mathrm{m}$ (bottom). (B) Images and graphs of reverse-transcription polymerase chain reaction and Western blot analyses of the relative expression of caspase- $3 \mathrm{mRNA}$ and protein. Results are presented as mean \pm standard error of the mean. ${ }^{*} \mathrm{P}<0.01$ compared with the sham-operated group. ${ }^{\dagger \dagger} \mathrm{P}<0.01$ compared with the $\mathrm{I} / \mathrm{R}$ group.

effect was not statistically significant. Western blotting analysis also showed that cleaved caspase-3 expression was higher in I/Rsubjected rats than in sham-operated rats; metformin or sildenafil treatment significantly reduced the $\mathrm{I} / \mathrm{R}$-induced increase in cleaved caspase-3 levels.

\section{DISCUSSION}

A variety of medicinal agents that inhibit oxidative stress and cellular inflammation protect the bladder against ROS insults following I/R injuries in animal models [21]. For example, metformin reduces ROS activity and restores ROS-induced vascular dysfunction [22], counteracts increases in MDA levels and ROS production, and ameliorates decreases in antioxidative substrates $[23,24]$. In a bladder I/R model, administration of sildenafil was found to increase oxygen saturation and bladder compliance, leading to restoration of microcirculation in the bladder wall [25]. Sildenafil may also have anti-inflammatory effects, inhibiting the production of ROS and proinflammatory enzymes [26]. We found that metformin and/or sildenafil re- 
stored SOD activity and MDA and MPO levels in a rat model of $\mathrm{I} / \mathrm{R}$-induced bladder injury. Thus, treatment with metformin or sildenafil may reduce I/R-induced oxidative stress and inflammation in bladder tissue, an effect related to the suppression of ROS activity.

MAPKs are important components of a physiologically important intracellular signaling pathway. MAPKs are important for cell survival and apoptosis; therefore, the control of these pathways is paramount in determining cell fate [27]. MAPKs can be activated by various stimuli. In general, ERK is activated in association with growth factors, and JNK and p38 MAPK respond to stress stimuli. These members of the MAPK family are not independent and have interactions that either stimulate or inhibit each other in cellular systems [28]. ROS can induce or regulate the activation of MAPK signaling pathways [29], and a cellular stimulus that induces ROS production can also activate MAPK signaling pathways in various cell types [30]. However, the mechanisms by which ROS activate MAPK signaling pathways in bladder $\mathrm{I} / \mathrm{R}$ injuries are not well understood. In the present study, we observed that I/R-induced injuries downregulated ERK expression and upregulated JNK and p38 expression, and that these changes were reversed after metformin and/or sildenafil treatment. However, coadministration of metformin and sildenafil was not more effective than either alone in activating MAPKs. Few studies have investigated drug interactions between metformin and PDE5 inhibitors. Sildenafil attenuated, not enhanced, the vaso-relaxant effect of metformin and completely blocked the vaso-relaxant effects of low concentrations of metformin [31]. Pharmacokinetic results showed that metformin and udenafil each competitively inhibited the metabolism of the other drug via hepatic cytochrome p450 3A1/2 [32]. Thus, our coadministration results may be associated with interactions between these drugs that inhibit metabolism.

Apoptosis is regulated by various molecular factors, including Bax and Bcl-2. Bax, a proapoptotic antagonist of Bcl-2 expression, is a $\mathrm{Bcl}-2$ binding protein, whereas $\mathrm{Bcl}-2$ inhibits apoptosis and prolongs cell survival [33]. In our study, the Bax/ Bcl-2 ratio paralleled the activation of JNK and p38 MAPK. The increased ratio resulting from $\mathrm{I} / \mathrm{R}$ injuries was ameliorated by administration of metformin and/or sildenafil. Metformin and sildenafil may inhibit apoptosis through activation of MAPK signaling pathways by reducing the $\mathrm{Bax} / \mathrm{Bcl}-2$ ratio. In addition, ROS activity has been shown to enhance NF- $\mathrm{kB}$ activation. Activated NF- $\mathrm{KB}$ increases the expression of genes en- coding inflammation-related transcripts and may play a key role in neutrophil accumulation during reperfusion injury. Metformin has been reported to inhibit NF- $\mathrm{KB}$ through activation of AMPK. Metformin also suppresses the expression of proinflammatory molecules by blocking the expression of NF$\kappa \mathrm{B}$ in vascular cells [34]. Our study found that $\mathrm{I} / \mathrm{R}$ injuries in the bladder increased NF- $\mathrm{KB}$ activity, indicating that $\mathrm{I} / \mathrm{R}$ injuries enhance cellular inflammation. The increase in NF- $\mathrm{kB}$ activity was reversed by treatment with metformin and/or sildenafil, suggesting that inhibition of NF- $\mathrm{kB}$ activity may play an important role in mediating bladder $\mathrm{I} / \mathrm{R}$ injury. In addition, the $\mathrm{I} / \mathrm{R}$-induced increase in NF- $\mathrm{BB}$ activity was reduced significantly more by metformin than by sildenafil, demonstrating the anti-inflammatory action of metformin.

In this study, we showed that metformin and sildenafil protected the bladder against I/R-induced injuries by inhibiting ROS production via activation of the MAPK and $\mathrm{Bax} / \mathrm{Bcl}-2$ signaling pathways, as well as by suppressing inflammation via inhibition of NF- $\mathrm{kB}$ activity. Moreover, metformin had a significantly greater effect on the MAPK signaling pathway and NF$\kappa \mathrm{B}$ activity than sildenafil, while the effects of these 2 drugs on oxidative stress did not differ. Coadministration of metformin and sildenafil was not more effective than administration of either drug alone.

\section{SUPPLEMENTARY MATERIALS}

Supplementary Tables 1 and 2 can be found via https://doi.org/ 10.5213/inj.2142206.103.

\section{AUTHOR CONTRIBUTION STATEMENT}

- Conceptualization: JHS, YGN

- Data curation: JMP, SWY, JYL, JSL, KHS

- Formal analysis: $S W Y, J Y L$

- Funding acquisition: GHK, YGN

- Methodology: JSL, KHS

- Project administration: JMP, JHS, YGN

- Visualization: JMP, GHK

- Writing-riginal draft: JMP, JHS

- Writing-review \& editing: JMP, JHS, YGN

\section{ORCID}

Jong Mok Park

0000-0002-4430-7851 


$\begin{array}{ll}\text { Ju Hyun Shin } & 0000-0001-5996-5876 \\ \text { Seung Woo Yang } & 0000-0002-8733-6564 \\ \text { Ji Yong Lee } & 0000-0003-1806-4750 \\ \text { Chung Lyul Lee } & 0000-0002-7157-4883 \\ \text { Jae Sung Lim } & 0000-0003-4324-8148 \\ \text { Ki Hak Song } & 0000-0002-6168-3664 \\ \text { Gun Hwa Kim } & 0000-0003-0360-4435 \\ \text { Yong Gil Na } & 0000-0002-0794-5459\end{array}$

\section{REFERENCES}

1. Suh J, Oh SJ, Cho SY. Comprehensive review of effective application of questionnaires for clinical research on lower urinary tract symptoms with translation and cultural adaptation to the Korean language. Int Neurourol J 2020;24:313-23.

2. Kim DK, Lee JY, Jung JH, Kim JH, Hah YS, Hong CH, et al. Alpha-1 adrenergic receptor blockers for the treatment of lower urinary tract symptoms in women: a systematic review and metaanalysis. Int Neurourol J 2019;23:56-68.

3. Bratslavsky G, Kogan BA, Matsumoto S, Aslan AR, Levin RM. Reperfusion injury of the rat bladder is worse than ischemia. J Urol 2003;170:2086-90.

4. Masuda H, Kihara K, Saito K, Matsuoka Y, Yoshida S, Chancellor $\mathrm{MB}$, et al. Reactive oxygen species mediate detrusor overactivity via sensitization of afferent pathway in the bladder of anaesthetized rats. BJU Int 2008;101:775-80.

5. Liochev SI. Reactive oxygen species and the free radical theory of aging. Free Radic Biol Med 2013;60:1-4.

6. Mirmiranpour H, Mousavizadeh M, Noshad S, Ghavami M, Ebadi $\mathrm{M}$, Ghasemiesfe $\mathrm{M}$, et al. Comparative effects of pioglitazone and metformin on oxidative stress markers in newly diagnosed type 2 diabetes patients: a randomized clinical trial. J Diabetes Complications 2013;27:501-7.

7. Zhou G, Myers R, Li Y, Chen Y, Shen X, Fenyk-Melody J, et al. Role of AMP-activated protein kinase in mechanism of metformin action. J Clin Invest 2001;108:1167-74.

8. Ling S, Xie H, Yang F, Shan Q, Dai H, Zhuo J, et al. Metformin potentiateds the effet of arsenic trioxide suppressing intrahepatic cholangiocarcinoma: roles of p38 MAPK, ERK3, and mTORC 1. J Hematol Oncol 2017;10:59.

9. Yuan J, Dong X, Yap J, Hu J. The MAPK and AMPK signalings: interplay and implication in targeted cancer therapy. J Hematol Oncol 2020;13:113.

10. Nesti L, Natali A. Metformin effects on the heart and the cardiovascular system: a review of experimental and clinical data. Nutr
Metab Cardiovasc Dis 2017;27:657-69.

11. Wang ZS, Liu XH, Wang M, Jiang GJ, Qiu T, Chen ZY, et al. Metformin attenuated the inflammation after renal ischemia/reperfusion and suppressed apoptosis of renal tubular epithelial cell in rats. Acta Cir Bras 2015;30:617-23.

12. Reffelmann T, Kloner RA. Phosphodiesterase 5 inhibitors: are they cardio-protective? Cardiovasc Res 2009;83:204-12.

13. Sohotnik R, Nativ O, Abbasi A, Awad H, Frajewicki V, Bishara B, et al. Phosphodiesterase-5 inhibition attenuates early renal ischemiareperfusioninduced acute kidney injury: assessment by quantitative measurement of urinary NGAL and KIM-1. Am J Physiol Renal Physiol 2013;304:1099-104.

14. Morsy MA, Ibrahim SA, Amin EF, Kamel MY, Rifaai RA, Hassan MK. Sildenafil ameliorates gentamicin-induced nephrotoxicity in rats: role of iNOS and eNOS. J Toxicol 2014;2014:489382.

15. Gotoh D, Torimoto K, Tatsumi Y, Hori S, Yamada A, Miyake M, et al. Tadalafil, a phosphodiesterase type 5 inhibitor, improves bladder blood supply and restores the initial phase of lower urinary tract dysfunction in diabetic rats. Neurourol Urodyn 2018;37:666-72.

16. Tzanavari T, Varela A, Theocharis S, Ninou E, Kapelouzou A, Cokkinos DV, et al. Metformin protects against infection-induced myocardial dysfunction. Metabolism 2016;65:1447-58.

17. Ozgur BC, Telli O, Yuceturk CN, Sarici H, Ozer E, Surer H, et al. The effect of sildenafil and udenafil on testicular damage following ischemia-reperfusion injury in rats. J Urol 2014;192:1272-7.

18. Durak I, Yurtarslanl Z, Canbolat O, Akyol O. A methodological approach to superoxide dismutase (SOD) activity assay based on inhibition of nitroblue tetrazolium (NBT) reduction. Clin Chim Acta 1993;214:103-4.

19. Wasowicz W, Nève J, Peretz A. Optimized steps in fluorometric determination of thiobarbituric acid reactive substances in serum: importance of extraction $\mathrm{pH}$ and influence of sample preservation and storage. Clin Chem 1993;39:2522-6.

20. Yang CH, Tsai PS, Wang TY, Huang CJ. Dexmedetomidine-ketamine combination mitigates acute lung injury in haemorrhagic shock rats. Resuscitation 2009;80:1204-10.

21. Levin RM, Xia L, Wei W, Schuler C, Leggett RE, Lin AD. Effects of ganoderma lucidum shell-broken spore on oxidative stress of the rabbit urinary bladder using an in vivo model of ischemia/reperfusion. Mol Cell Biochem 2017;435:25-35.

22. Rosen P, Wiernsperger NF. Metformin delays the manifestation of diabetes and vascular dysfunction in Goto-Kakizaki rats by reduction of mitochondrial oxidative stress. Diabetes Metab Res Rev 2006;22:323-30.

23. Taheri N, Azarmi Y, Neshat M, Garjani A, Doustar Y. Study the ef- 
fects of metformin on renal function and structure after unilateral ischemia-reperfusion in rat. Res Pharm Sci 2012;7:77.

24. Sung JY, Choi HC. Metformin-induced AMP-activated protein kinase activation regulates phenylephrine-mediated contraction of rat aorta. Biochem Biophys Res Commun 2012;421:599-604.

25. Scheepe JR, Amelink A, Wolffenbuttel KP, Kok DJ. Influence of sildenafil on blood oxgen saturation of the obstructed bladder. BMC Urol 2014;14:44.

26. Koupparis AJ, Jeremy JY, MuzaVar S, Persad R, Shukla N. Sildenafil inhibits the formation of superoxide and the expression of gp 47 $\mathrm{NAD}[\mathrm{P}] \mathrm{H}$ oxidase induced by the thromboxane A2 mimetic, U46619, in corpus cavernosal smooth muscle cells. BJU Int 2005; 96:423-7.

27. Plotnikov A, Zehorai E, Procaccia S, Seger R. The MAPK cascades: Signaling components, nuclear roles and mechanisms of nuclear translocation. Biochim Biophys Acta 2011;1813:1619-33.

28. Son Y, Kim S, Chung HT, Pae HO. Reactive oxygen species in the activation of MAP kinases. Methods Enzymol 2013;528:27-48.

29. McCubrey JA, Lahair MM, Franklin RA. Reactive oxygen speciesinduced activation of the MAP kinase signaling pathways. Antiox- id Redox Signal 2006;8:1775-89.

30. Shahid M, Kim M, Yeon A, Jin P, Kim WK, You S, et al. Pioglitazone alters the proteomes of normal bladder epithelial cells but shows no tumorigenic effects. Int Neurourol J 2020;24:29-40.

31. Shin MS, Kim TW, Park SS, Ko IG, Kim CJ, Kim M, et al. Longterm surgical and chemical castration deteriorates memory function through downregulation of PKA/CREB/BDNF and c-Raf/ MEK/ERK pathways in hippocampus. Int Neurourol J 2019;23: 116-24.

32. Choi YH, Chung SJ, Lee MG. Pharmacokinetic interaction between DA-8159, a new erectogenic, and metformin in rats: competitive inhibition of metabolism via hepatic CYP3A1/2. Br J Phamacol 2008;153:1568-78.

33. Rho JH, Ko IG, Jin JJ, Hwang L, Kim SH, Chung JY, et al. Polydeoxyribonucleotide ameliorates inflammation and apoptosis in achilles tendon-injury rats. Int Neurourol J 2020;24:79-87.

34. Hattori Y, Suzuki K, Hattori S, Kasai K. Metformin inhibits cytokine-induced neclear factor kappaB activation via AMP-activated protein kinase activation in vascular endothelial cells. Hypertension 2006;47:1183-8. 